\title{
Analysis of Image Compression Using Wavelet
}

\section{Amar Nath Gupta ${ }^{1}$, Bikash Chandra Sahana ${ }^{2}$, Vijay Kumar Anand ${ }^{3}$ (Dept. of ECE, NIT Patna, India)}

Abstract : Recently, wavelet has a powerful tool for image compression. This paper analysis the mean square error, peak signal to noise ratio and bit-per-pixel ratio of compressed image with different decomposition level by using wavelet.

Keywords - Image, wavelet, BPP, PSNR, MSE

\section{INTRODUCTION}

Image is a two dimensional function $\mathrm{f}(\mathrm{x}, \mathrm{y})$, where $\mathrm{x}$ and $\mathrm{y}$ are spatial(plane) coordinates, and the amplitude of $f$ at any pair of coordinates $(x, y)$ is called the intensity of the image at that point. Wavelets are a fairly simple mathematical tool that cuts up data or functions into different frequency components and then studies each component with a resolution matched to its scale [1]. The wavelet is a powerful tool which gives the spatial and frequency characteristics of an image, on the other hand, Fourier transform gives only frequency characteristics of an image [2].

Now beginning from a given image, the aim of compression is to reduced the number of bits needed to represents it, while storing information of acceptable quality. The effective solution for this problem is wavelet. The complete chain of compression includes iterative phases of quantization, coding and decoding, in addition to the wavelet processing itself [3].

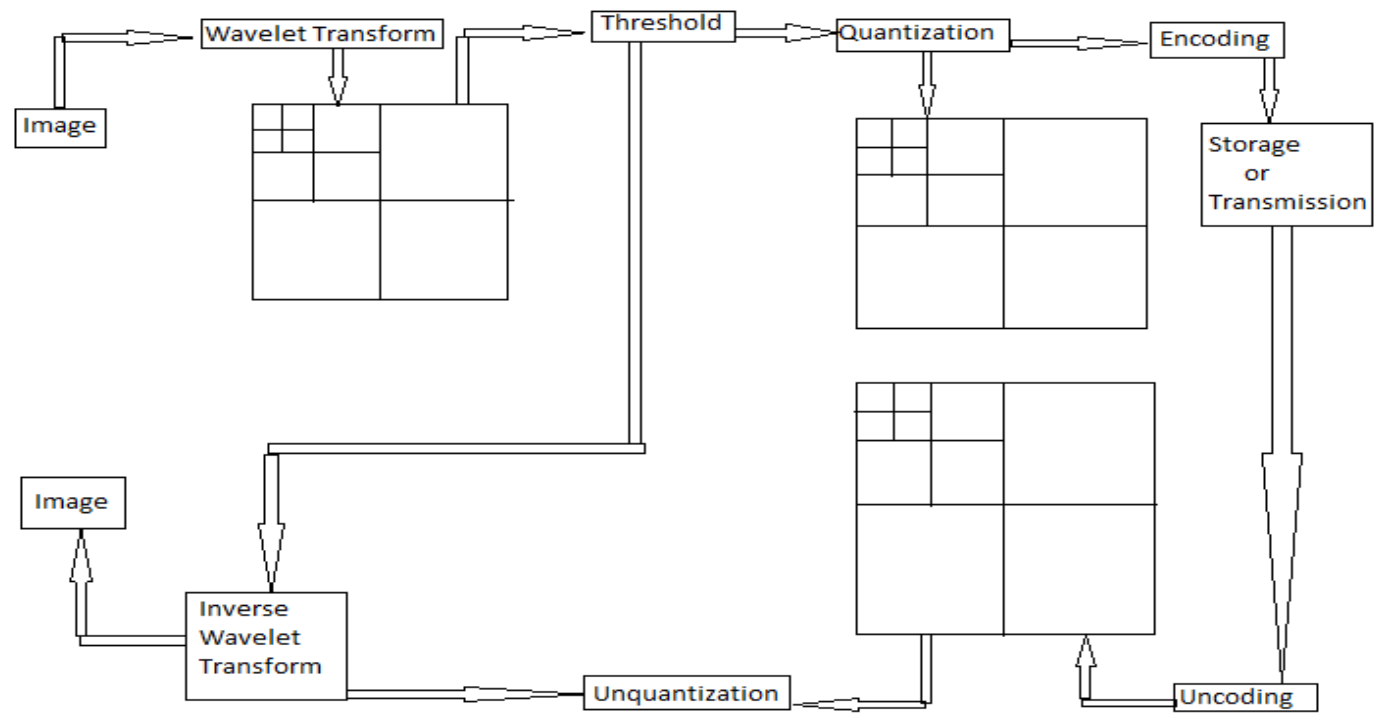

\section{A. Compression performance}

\section{BACKGROUND \& EQUATIONS}

Two quantitative measures giving equivalent information are commonly used as a performance indicator for the compression:

The compression ratio $\mathrm{CR}$, which means that the compressed image is stored using only CR\% of the initial storage size.

The Bit-per-pixel ratio BPP, which gives the number of bits required to store one pixel of the image.

\section{B. Perceptual Quality}

Two measures are commonly used to evaluate the perceptual quality:- 
The Mean Square Error (MSE).It represents the mean squared error between the compressed and the original image and is given by

$$
M S E=\frac{1}{m n} \sum_{i=0}^{m=1} \sum_{j=0}^{n=1}\left|x(i, j)-x_{c}(i, j)\right|^{2}
$$

The Peak Signal to Noise Ratio (PSNR).It represents a measure of the peak error and is expressed in decibels. It is defined by:

$$
P S N R=10 \log _{10} \frac{255^{2}}{M S E}
$$

SPIHT Algorithm:-SPIHT algorithm was introduced by Said [4] and Pearlman [5].It is a simple, efficient and powerful compression algorithm. This algorithm gives the idea to compute the highest PSNR value of a variety of images. The important feature of this algorithm is that at any point during the decoding of an image, the quality of the displayed image is the best that can be achieved for the number of bits input by the decoder up to that moment.

The wavelet coefficients can be referred as $c_{i, j}$. In a progressive transmission method, the decoder starts by setting the reconstruction image to zero. It then inputs (encoded) transform coefficients, decodes them and uses them to generate an improved reconstruction image. The main aim in progressive transmission is to transmit the most important image information first. SPIHT uses the mean square error (MSE) distortion measure.

SPIHT Coding:-It is important to have the encoder and decoder test sets for significance in the same way, so the coding algorithm uses three lists called list of significance pixels (LSP), list of insignificant pixels (LIP), and list of insignificant sets (LIS).

1. Intialization: Set $n$ to $\left[\log _{2} \max _{\mathrm{i}, \mathrm{j}}\left(\mathrm{c}_{\mathrm{i}, \mathrm{j}}\right)\right]$ and transmit $\mathrm{n}$. Set the LSP to empty. Set the LIP to the coordinates of all the roots $(\mathrm{i}, \mathrm{j}) \in \mathrm{H}$. Set the LIS to the coordinates of all the roots $(\mathrm{i}, \mathrm{j}) \in \mathrm{H}$ that have descendents.

2. Sorting pass:

2.1 for each entry $(i, j)$ in the LIP do:

2.1.1 output $S_{n}(i, j)$;

2.1.2 if $S_{n}(i, j)=1$, move (i,j) to the LSP and output the sign of $c_{i, j}$;

2.2 for each entry $(i, j)$ in the LIS do:

2.2.1 if the entry is of type $A$, then

Output $S_{n}(D(i, j))$; then

For each $(k, 1) \in \mathrm{O}(\mathrm{i}, \mathrm{j})$ do:

Output $S_{n}(k, 1)$;

If $S_{n}(k, 1)=1$, add $(k, 1)$ to the LSP,

Output the sign of $c_{k, 1}$;

If $\mathrm{S}_{\mathrm{n}}(\mathrm{k}, 1)=0$, append $(\mathrm{k}, 1)$ to the

LIP;

If $\mathrm{L}(\mathrm{i}, \mathrm{j})$ is not equal to 0 , move (i,j) to the end of the LIS, as a type-B entry and go to step 2.2.2; else, remove entry $(\mathrm{i}, \mathrm{j})$ from the LIS;

2.2.3 if the entry is of type $B$, then

Output $S_{n}(L(i, j))$;

If $S_{n}(L(I, j))=1$, then

append each $(\mathrm{K}, 1) \in \mathrm{O}(\mathrm{i}, \mathrm{j})$ to the LIS as a type

A entry:

Remove (i,j) from the LIS:

3. Refinement pass: for each entry (i,j) in the LSP, except those included in the last sorting pass (the one with the same $n$ ), output the nth most significant bit of $\left|c_{i j}\right|$;

4. Loop; decrement $\mathrm{n}$ by 1 and go to step 2 if needed.

EZW Algorithm:- EZW algorithm is the powerful algorithm based on wavelet based image compression. The other algorithm is generated by the help of EZW algorithm.EZW algorithm was introduced by Shapiro [6].EZW means embedded zerotree wavelet. EZW approximates higher frequency coefficients of a wavelet transformed image. The EZW algorithm is as follow:

1. Initialization: Set the threshold $T$ to the smallest power of 2 that is greater than $\max (i, j) \mid c_{i, j} / 2$, where $c_{i, j}$ are the wavelet coefficients.

2. Significance map coding: Scan all the coefficients in a predefined way and output a symbol when $\left|c_{i, j}\right|>T$, when the decoder inputs this symbol, it set sets $c_{i j}= \pm 1.5 \mathrm{~T}$ 
3. Refinement: Refine each significant coefficient by sending one more bit of its binary representation. When the decoder receives this, it increments the current coefficient value by $\pm 0.25 \mathrm{~T}$

4. Set $\mathrm{T}=\mathrm{T} / 2$, and go to step 2 if more iterations are needed.

EZW coding: A wavelet coefficient $c_{i, j}$ is considered insignificant with respect to the current threshold $\mathrm{T}$ if $\left|\mathrm{c}_{\mathrm{i}, \mathrm{j}}\right|=\mathrm{T}$. The zerotree data structure can be constructed from the following experimental result: If a wavelet coefficients at a coarse scale (i.e., high in the image pyramid) is insignificant with respect to a given threshold $\mathrm{T}$, then all of the coefficients of the same orientation in the same spatial location at finer scales (i.e., located lower in the pyramid) are very likely to be insignificant with respect to T. In each iteration, all the coefficients are scanned in the order. This guarantees that when a node is visited, all its parents will already have been scanned.

Each coefficients visited in the scan is classified as a zerotree (ZTR), an isolated zero (IZ), positive significant (POS), or negative significant (NEG).A zerotree root is a coefficient that is insignificant and all its descendants ( in the same spatial orientation tree) are also insignificant. Such a coefficient becomes the root of a zerotree. It is encoded with a special symbol (denoted by ZTR). When the decoder inputs a ZTR symbol.

\section{SimUlation RESUlT}

The image compression techniques discussed in section II were applied to a variety of images using Matlab® software.

Lena, Barbara and boat image $(512 * 512)$
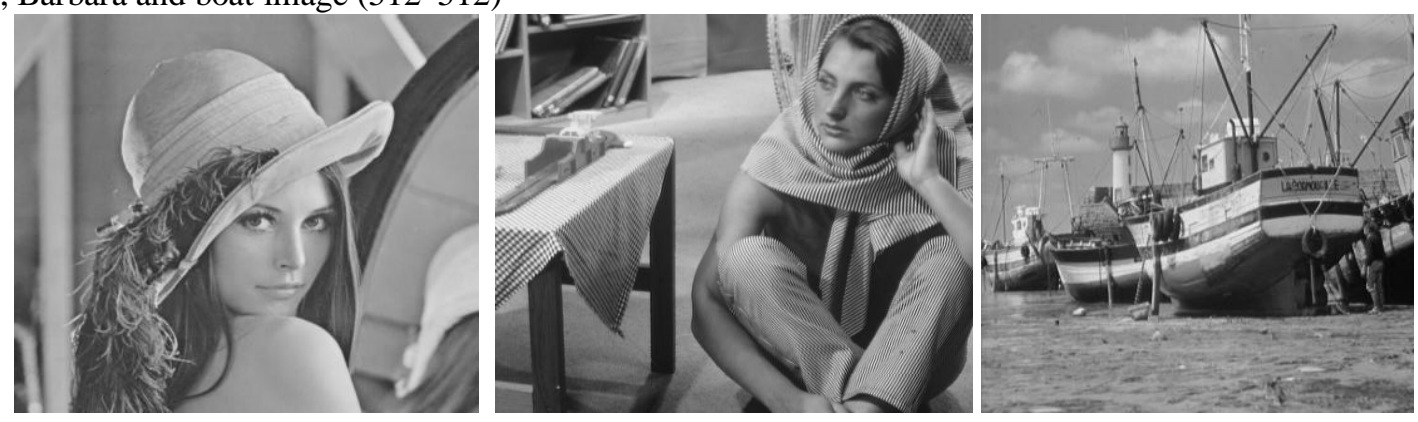

Table:-

\begin{tabular}{|c|c|c|c|c|c|c|}
\hline $\begin{array}{l}\text { Image } \\
\text { Type }\end{array}$ & Wavelet & $\begin{array}{l}\text { Compression } \\
\text { Parameter }\end{array}$ & $\begin{array}{l}\text { Decomposition } \\
\text { Level }\end{array}$ & PSNR & MSE & BPP \\
\hline \multirow{10}{*}{$\begin{array}{l}\text { Lena.bmp } \\
(512 * 512)\end{array}$} & \multirow[t]{10}{*}{ Haar } & \multirow{5}{*}{$\begin{array}{l}\text { EZW, } \\
\mathrm{Nb} \\
\text { Encoding } \\
\text { Loops, } 8\end{array}$} & 1 & 51.72 & 0.4376 & 7.7551 \\
\hline & & & 2 & 44.55 & 2.282 & 3.6219 \\
\hline & & & 3 & 39.72 & 6.938 & 1.7131 \\
\hline & & & 4 & 35.74 & 17.35 & 0.83084 \\
\hline & & & 5 & 32.03 & 40.78 & 0.38043 \\
\hline & & \multirow{5}{*}{$\begin{array}{l}\text { SPIHT, } \\
\mathrm{Nb} \\
\text { Encoding } \\
\text { Loops, } 8\end{array}$} & 1 & 37.65 & 11.16 & 7.0175 \\
\hline & & & 2 & 36.95 & 13.11 & 2.3537 \\
\hline & & & 3 & 34.15 & 25 & 0.87985 \\
\hline & & & 4 & 31.12 & 50.23 & 0.34708 \\
\hline & & & 5 & 28.1 & 100.6 & 0.13486 \\
\hline
\end{tabular}

\begin{tabular}{|c|c|c|c|c|c|c|}
\hline Image Type & Wavelet & $\begin{array}{l}\text { Compression } \\
\text { Parameter }\end{array}$ & $\begin{array}{l}\text { Decomposition } \\
\text { Level }\end{array}$ & PSNR & MSE & BPP \\
\hline \multirow{10}{*}{$\begin{array}{l}\text { barbara.bmp } \\
(512 * 512)\end{array}$} & \multirow[t]{10}{*}{ Haar } & \multirow{5}{*}{$\begin{array}{l}\text { EZW, } \\
\mathrm{Nb} \\
\text { Encoding } \\
\text { Loops, } 8\end{array}$} & 1 & 53.18 & 0.3126 & 8.9462 \\
\hline & & & 2 & 45.48 & 1.843 & 5.2842 \\
\hline & & & 3 & 39.54 & 7.234 & 3.1143 \\
\hline & & & 4 & 34.18 & 24.86 & 1.7453 \\
\hline & & & 5 & 28.95 & 82.86 & 0.81506 \\
\hline & & \multirow{5}{*}{$\begin{array}{l}\text { SPIHT, } \\
\mathrm{Nb} \\
\text { Encoding } \\
\text { Loops, } 8\end{array}$} & 1 & 37.49 & 11.59 & 7.8746 \\
\hline & & & 2 & 35.59 & 17.94 & 3.2138 \\
\hline & & & 3 & 32.21 & 39.05 & 1.4844 \\
\hline & & & 4 & 27.83 & 107.1 & 0.66656 \\
\hline & & & 5 & 24.24 & 245.2 & 0.23734 \\
\hline
\end{tabular}


Analysis of image compression using wavelet

\begin{tabular}{|c|c|c|c|c|c|c|}
\hline $\begin{array}{l}\text { Image } \\
\text { Type }\end{array}$ & Wavelet & $\begin{array}{l}\text { Compression } \\
\text { Parameter }\end{array}$ & $\begin{array}{l}\text { Decomposition } \\
\text { Level }\end{array}$ & PSNR & MSE & BPP \\
\hline \multirow{12}{*}{$\begin{array}{l}\text { boat.bmp } \\
(512 * 512)\end{array}$} & \multirow[t]{12}{*}{ Haar } & \multirow{5}{*}{$\begin{array}{l}\text { EZW, } \\
\text { Nb Encoding } \\
\text { Loops, } 8\end{array}$} & 1 & 53.37 & 0.2993 & 9.169 \\
\hline & & & 2 & 44.94 & 2.085 & 5.0627 \\
\hline & & & 3 & 38.34 & 9.534 & 2.6319 \\
\hline & & & 4 & 33.8 & 27.13 & 1.2751 \\
\hline & & & 5 & 30.1 & 63.54 & 0.58871 \\
\hline & & \multirow{7}{*}{$\begin{array}{l}\text { SPIHT, } \\
\text { Nb Encoding } \\
\text { Loops, } 8\end{array}$} & 1 & 38.05 & 10.19 & 7.705 \\
\hline & & & 2 & 35.51 & 18.29 & 2.9371 \\
\hline & & & 3 & 32.36 & 37.81 & 1.1774 \\
\hline & & & 4 & 29.16 & 78.93 & 0.49246 \\
\hline & & & 5 & 26.05 & 161.5 & 0.19269 \\
\hline & & & 4 & 33.32 & 30.25 & 1.4442 \\
\hline & & & 5 & 29.8 & 68.16 & 0.6683 \\
\hline
\end{tabular}

Graph:-

PSNR Vs Decomposition level using Haar Wavelet
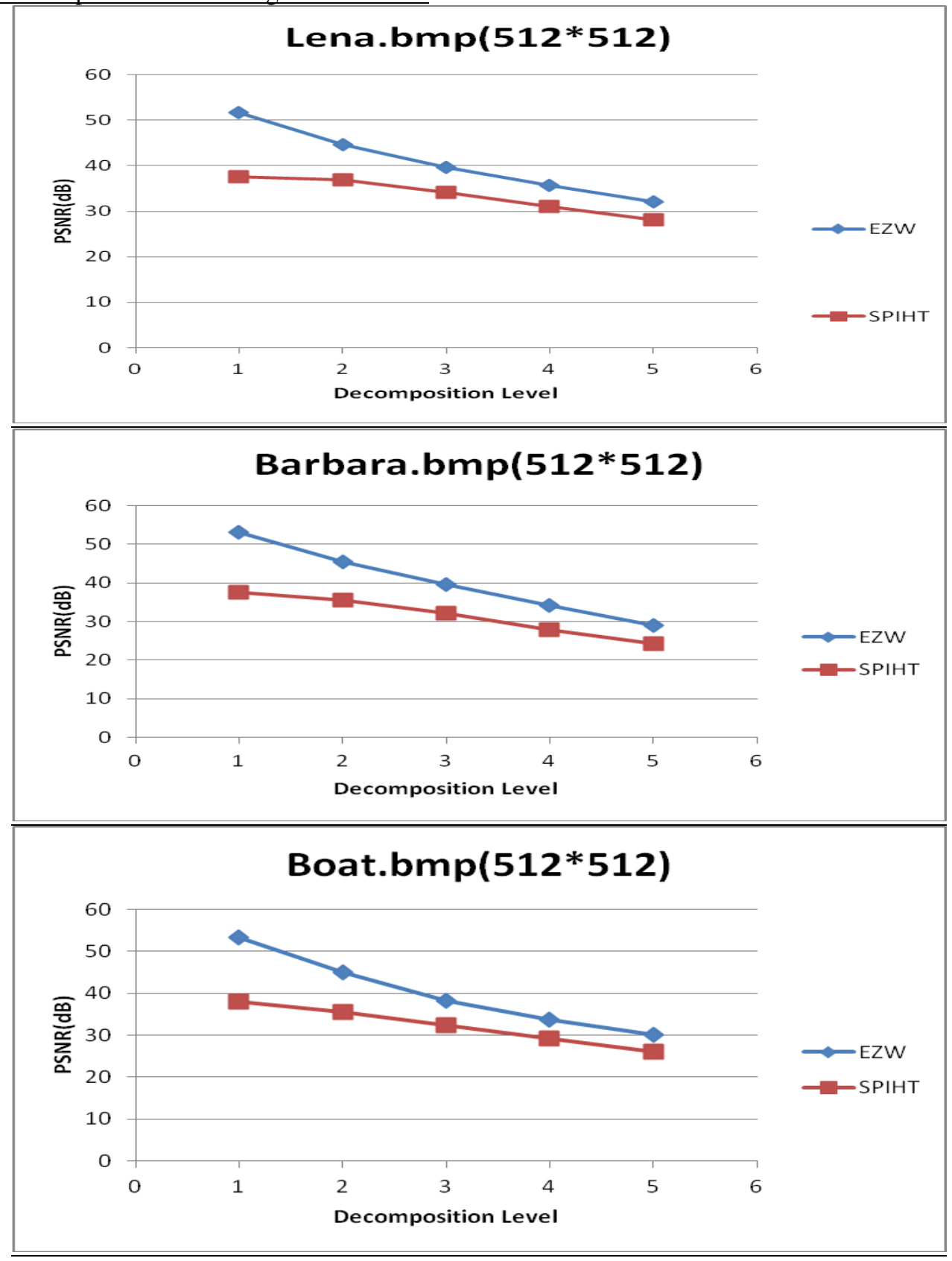
MSE VS Decomposition Level using Haar wavelet

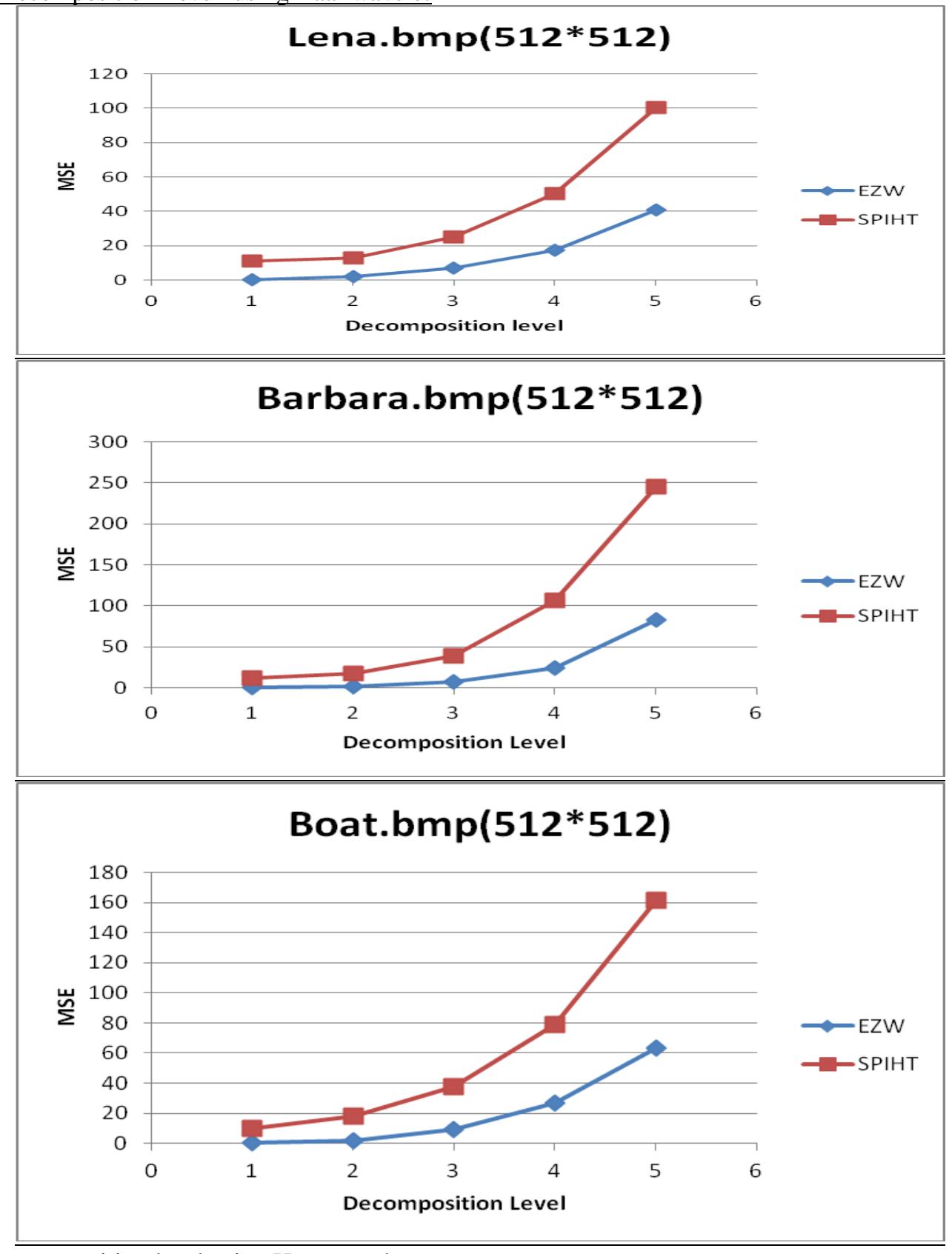

BPP VS Decomposition level using Haar wavelet

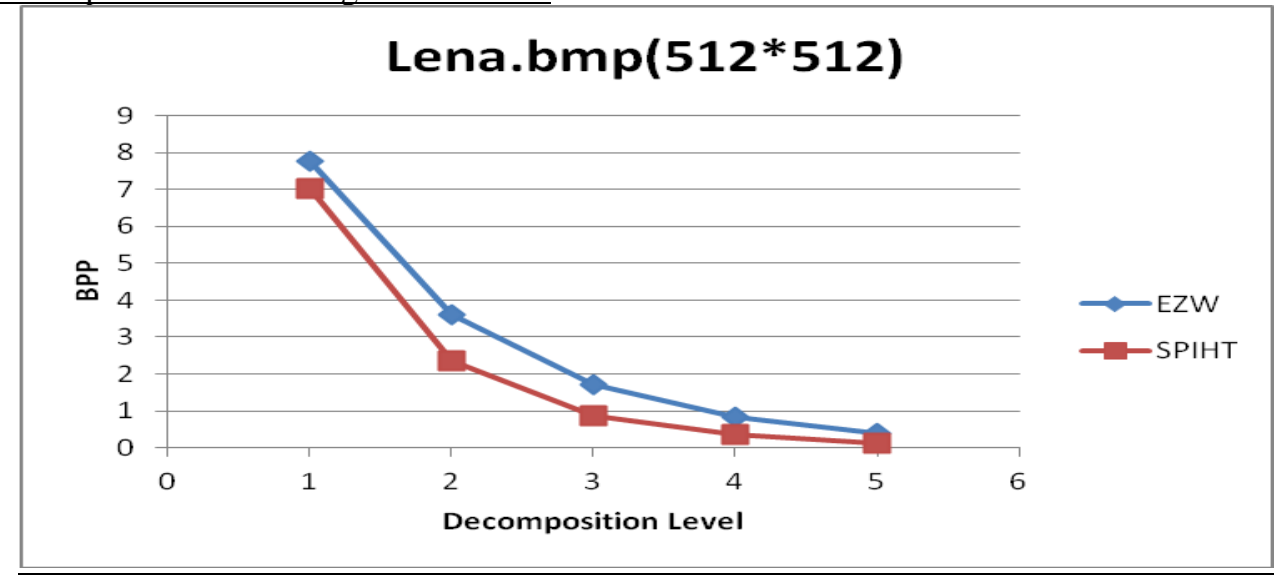




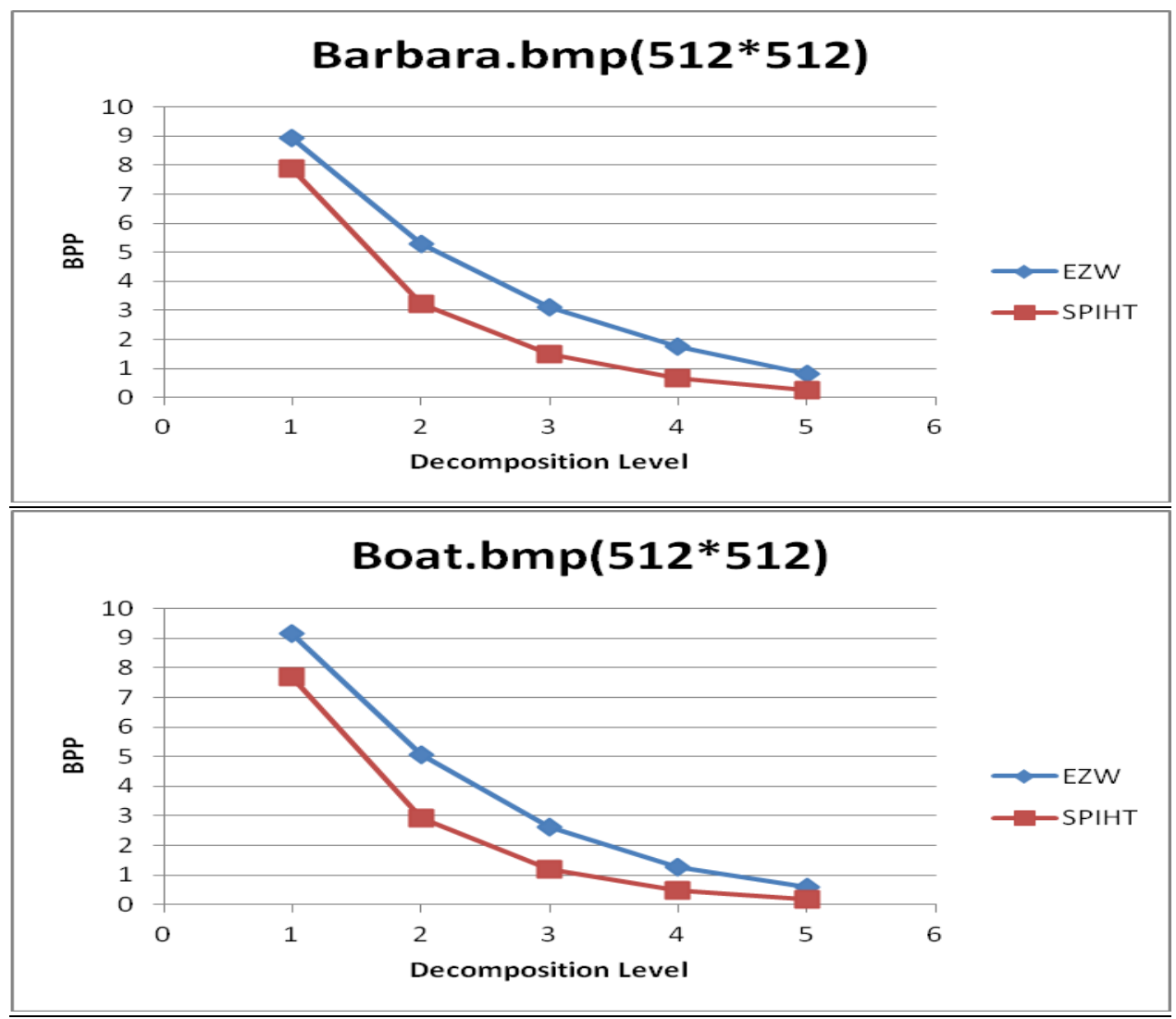

\section{CONCLUSION}

We see that higher the PSNR value gives the better the quality of the compressed or reconstructed image. Higher the MSE values degrade the quality of the compressed or reconstructed image. Also, increasing the decomposition level Bit-per-pixel ratio decreases.

\section{REFERENCES}

[1] Ingrid Daubechies, ten lectures on wavelets, (CBMS-NSF regional conference series in applied mathematics, 1992.$)$

[2] Rafael C. Gonzalez, Richard E. Woods, Steven L. Eddins, digital image processing using matlab, (Pearson Education, 2004.)

[3] The Mathworks Inc., Wavelet Toolbox User Guide, Natick, MA, 2011.

[4] A. Said, W.A. Pearlman, image compression using the spatial-orientation tree, IEEE Int. Symp. on circuits and systems, Chicago, II, PP.279-282,1993

[5] A. Said, W.A. Pearlman, a new, fast, and efficient image codec based on set partitioning in hierarchical trees, IEEE trans. on circuits and systems for video technology, vol.6,No.3,pp.243-250,1996

[6] J.M. Shapiro, embedded image coding using zerotrees of wavelet coefficients, IEEE Trans. Signal Proc., vol.41,No.12,pp.34453462,1993 